\title{
BOTT PERIODICITY AND THE PARALLELIZABILITY OF THE SPHERES
}

\author{
By M. F. ATIYAH AND F. HIRZEBRUCH
}

Received 12 April 1960

Introduction. The theorems of Bott(4), (5) on the stable homotopy of the classical groups imply that the sphere $S^{n}$ is not parallelizable for $n \neq 1,3,7$. This was shown independently by Kervaire(8) and Milnor(7), (9). Another proof can be found in (3), $\$ 26 \cdot 11$. The work of J. F. Adams (on the non-existence of elements of Hopf invariant one) implies more strongly that $S^{n}$ with any (perhaps extraordinary) differentiable structure is not parallelizable if $n \neq 1,3,7$. Thus there exist already four proofs for the non-parallelizability of the spheres, the first three mentioned relying on the Bott theory, as given in (4), (5). The purpose of this note is to show how the refined form of Bott's results given in (6) leads to a very simple proof of the non-parallelizability (only for the usual differentiable structures of the spheres). We shall prove in fact the following theorem due to Milnor (9) which implies the non-parallelizability.

THEOREm 1. There exists a real vector bundle $\xi$ over the sphere $S^{n}$ with $w_{n}(\xi) \neq 0$ only for $n=1,2,4$ or 8 .

$w_{i}(\xi) \in H^{i}\left(B_{\xi}, Z_{2}\right)$ denotes the $i$ th Stiefel-Whitney class of the real vector bundle $\xi$ with base $B_{\xi}$. We put $w(\xi)=\sum_{i=0}^{\infty} w_{i}(\xi)$.

Theorem 1 is a consequence of

Theorem 2. Let $Y$ be a finite $\mathrm{CW}$-complex, not necessarily connected. The (total) Stiefel-Whitney class $w(\eta)$ of any real vector bundle $\eta$ over the 9 -fold suspension of $Y$ equals 1 , i.e. $w_{i}(\eta)=0$ for $i>0$.

Theorem 2 takes care of the sphere $S^{n}$ for $n \geqslant 9$. Since the homotopy group $\pi_{r}\left(B_{o}\right)$ of the classifying space $B_{o}$ of the infinite orthogonal group vanishes for $r=3,5,6,7$ (see (5)), Theorem 1 is proved in these dimensions. We recall that $\pi_{1}\left(B_{O}\right)=Z_{2}, \pi_{2}\left(B_{O}\right)=Z_{2}, \pi_{4}\left(B_{O}\right)=Z$ and $\pi_{8}\left(B_{O}\right)=Z$. The generators of these groups correspond to the Hopf bundles over $S^{1}$ (Möbius), $S^{2}, S^{4}$ and $S^{8}$. For the Hopf bundle over $S^{r}(r=1,2,4,8)$, the Stiefel-Whitney class $w_{r} \in H^{r}\left(S^{r}, Z_{2}\right)$ is not zero.

By the Bott periodicity we shall calculate the Stiefel-Whitney classes of the real vector bundles over the eightfold suspension of a space $X$ by means of the StiefelWhitney classes of the real vector bundles over $X$ (see $\S 4$, Proposition). For this calculation (which has Theorem 2 as an immediate consequence) we use the tensor product description of the Bott periodicity (6) and the formula for the Stiefel-Whitney classes of the tensor product of two real vector bundles $(\S 3)$.

In this paper, we work in the class of finite $\mathrm{CW}$-complexes. This is much too strong a restriction, but is made for convenience. 
1. The spaces $X, Y, \ldots$ will be connected finite $\mathrm{CW}$-complexes except where otherwise mentioned. Let $K O(X)$ be the Grothendieck ring obtained from real vector bundles (1). The trivial vector bundle with fibre dimension 1 represents the unit of this ring. If $X$ has a base point $x_{0}$, then $\widetilde{K O}(X)$ is by definition the kernel of the ring homomorphism

$$
\epsilon: K O(X) \rightarrow K O\left(\left\{x_{0}\right\}\right)=\mathbf{Z} .
$$

Since $\varepsilon$ attaches to each vector bundle its fibre dimension, the homomorphism $\varepsilon$ does not depend on the choice of $x_{0}$ ( $X$ being connected). There is a direct sum decomposition

$$
K O(X)=\mathbf{Z} \oplus \widetilde{K O}(X),
$$

where the direct summand $Z$ represents the integral multiples of the unit 1 .

For spaces $X, Y$ with base points $x_{0}, y_{0}$, the spaces $X \vee Y$ and $X \wedge Y$ are defined. $X \vee Y$ is obtained from the (disjoint) topological sum of $X$ and $Y$ by identifying $x_{0}$ and $y_{0}$ to one point which becomes the base point of $X \vee Y$. The space $X \vee Y$ may be regarded as the subspace $X \times y_{0} \cup x_{0} \times Y$ of $X \times Y$, and $X \wedge Y$ is $X \times Y$ with $X \vee Y$ collapsed to a point which becomes the base point of $X \wedge Y$. We have the natural maps

$$
X \vee Y \rightarrow X \times Y \rightarrow X \wedge Y,
$$

and the corresponding split exact sequence (compare (2))

with

$$
\begin{gathered}
0 \rightarrow \widetilde{K O}(X \wedge Y) \stackrel{i}{\rightarrow} \widetilde{K O}(X \times Y) \stackrel{p}{\rightarrow} \widetilde{K O}(X \vee Y) \rightarrow 0, \\
\widetilde{K} O(X \vee Y) \cong \widetilde{K O}(X) \oplus \widetilde{K O}(Y) .
\end{gathered}
$$

For $a \in K O(X)$ and $b \in K O(Y)$, the tensor product $a \otimes b \in K O(X \times Y)$ is defined. If $a \in \widetilde{K O}(X)$ and $b \in \widetilde{K O}(Y)$, then $a \otimes b$ is in $\widetilde{K O}(X \times Y)$ and lies in the kernel of $p$. Therefore, by (2), the tensor product of $a \in \widetilde{K O}(X)$ and $b \in \widetilde{K O}(Y)$ gives an element of $\widetilde{K} O(X \wedge Y)$, also denoted by $a \otimes b$. (We consider $K O(X \wedge Y)$ as a subring of $K O(X \times Y)$.)

All this is analogous to ordinary cohomology theory. Let $H^{*}$ be the cohomology ring with coefficients in some ring with unit. Let $\tilde{H}^{*}$ be the ideal of $H^{*}$ consisting of the direct sum of the positive dimensional cohomology groups. Then for $a \in \tilde{H}^{*}(X)$ and $b \in \tilde{H}^{*}(Y)$, we have the tensor product $a \otimes b \in \tilde{H}^{*}(X \wedge Y)$. We regard $H^{*}(X \wedge Y)$ as a subring of $H^{*}(X \times Y)$.

Let $S^{n}$ be the $n$-sphere with base point. $S^{n} \wedge X=S^{n-1} \wedge S^{1} \wedge X$ is the $n$-fold suspension of $X$ (since $\wedge$ is associative). Let $g$ be the non-zero element of $H^{n}\left(S^{n}, Z_{2}\right)$. The suspension isomorphism

is given by

$$
\begin{aligned}
& \left.\begin{array}{l}
\delta^{n}: \tilde{H}^{*}\left(X, \mathbf{Z}_{2}\right) \rightarrow \tilde{H}^{*}\left(S^{n} \wedge X, \mathbf{Z}_{2}\right), \\
\delta^{n}: H^{i}\left(X, \mathbf{Z}_{2}\right) \rightarrow H^{n+i}\left(S^{n} \wedge X, Z_{2}\right) \quad(i>0),
\end{array}\right\} \\
& \delta^{n}(x)=g \otimes x, \quad x \in \tilde{H}^{*}\left(X, Z_{2}\right) .
\end{aligned}
$$

2. The (total) Stiefel-Whitney class satisfies the Whitney multiplication theorem $w(\xi \oplus \eta)=w(\xi) w(\eta)$, where $\xi$ and $\eta$ are real vector bundles over the same base space $X$. This implies easily that we have a natural homomorphism

$$
w: K O(X) \rightarrow 1+\sum_{i>0} H^{i}\left(X, Z_{2}\right)=G\left(X, \mathbf{Z}_{2}\right) .
$$


'Homomorphism' is meant with respect to the additive structure of $K O(X)$ and the commutative group structure of $G\left(X, Z_{2}\right)$ given by the cup-product. For any $x \in K O(X)$, the Stiefel-Whitney class $w_{i}(x) \in H^{i}\left(X, Z_{2}\right)$ is well defined.

3. Given two real vector bundles $\xi$ and $\eta$ over $X$ with fibres $\mathbf{R}^{m}$ and $\mathbf{R}^{n}$ respectively, the (total) Stiefel-Whitney class of their tensor product can be calculated in terms of $w(\xi)$ and $w(\eta)$, (see (3), §11). If we write 'formally'

then

$$
w(\xi)=\prod_{i=1}^{m}\left(1+x_{i}\right) \quad \text { and } \quad w(\eta)=\prod_{j=1}^{n}\left(1+y_{j}\right)
$$

This formula has to be interpreted as follows. Consider for a moment the $x_{i}$ and $y_{j}$ as indeterminates. Express the right-hand side of (6) as a polynomial in the elementary symmetric functions $a_{r}$ of the $x_{i}$ and $b_{s}$ of the $y_{j}$ with integral coefficients. Then replace $a_{r}$ by $w_{r}(\xi)$ and $b_{s}$ by $w_{s}(\eta)$ and reduce the coefficients mod 2 .

4. Let $\rho$ be the Hopf bundle over the sphere $S^{8}$. This is a real vector bundle with fibre $\mathbf{R}^{8}$. The Euler class of $\rho$ is a generator of $H^{8}\left(S^{8}, Z\right)$. The Stiefel-Whitney class $w(\rho)$ equals $1+g$ where $g$ is the non-zero element of $H^{8}\left(S^{8}, \mathbf{Z}_{2}\right)$. The bundle $\rho$ determines an element of $K O\left(S^{8}\right)$ which we also denote by $\rho$, the element $\rho-8$ belongs to $\widetilde{K O}\left(S^{8}\right)$, and $\rho-8$ is a generator of this infinite cyclic group.

Assume that $S^{8}$ and $X$ have been given base points. According to Bott (6) there is an additive isomorphism

$$
\beta: \widetilde{K O}(X) \rightarrow \widetilde{K O}\left(S^{8} \wedge X\right)
$$

which may be given as follows

$$
\beta(x)=(\rho-8) \otimes x, \quad \text { where } \quad x \in \widetilde{K O}(X) .
$$

We wish to calculate the Stiefel-Whitney class $w\{\beta(x)\}$ in terms of $w(x)$.

Proposition. Let $X$ be a connected finite $\mathrm{CW}$-complex, and $x \in \widetilde{K O}(X)$. Then

$$
w\{\beta(x)\}=1+g \otimes \sum_{k=1}^{\infty} s_{8 k}\left\{w_{1}(x), \ldots, w_{8 k}(x)\right\} \in H^{*}\left(S^{8} \wedge X, Z_{2}\right)
$$

where $g$ is the non-zero element of $H^{8}\left(S^{8}, \mathrm{Z}_{2}\right)$ and where $s_{r}\left(\delta_{1}, \ldots, \delta_{r}\right)$ is the polynomial with integral coefficients which expresses $x_{1}^{r}+\ldots+x_{n}^{r}(n \geqslant r)$ in terms of the elementary symmetric functions $\delta_{j}$ of the $x_{i}$.

Let us first see how Theorem 2 of the introduction is derived from this proposition. We put $S^{1} \wedge Y=X$. Thus $X$ is the suspension of $Y$. It is connected. Any real vector bundle $\eta$ (fibre $\mathrm{R}^{k}$ ) over $S^{8} \wedge X=S^{9} \wedge Y$ represents an element $\eta$ of $K O\left(S^{8} \wedge X\right)$. Then $\eta-k \in \widetilde{K O}\left(S^{8} \wedge X\right)$ and $w(\eta-k)=w(\eta)$. The element $\eta-k$ is, by (1), of the form $\beta(x), x \in \widetilde{K O}(X)$. We have to prove that $w\{\beta(x)\}=1$. But in $X=\mathcal{S}^{1} \wedge Y$ all products of cohomology classes of positive dimension vanish. The polynomial $s_{r}$ is of the form $(-1)^{r-1} r \delta_{r}+$ composite terms. Therefore $s_{8 k}\left\{w_{1}(x), \ldots, w_{8 k}(x)\right\}=-8 k w_{8 k}(x)=0 \bmod 2$.

5. Proof of the preceding proposition. By the classification theorem or by a more direct argument it is known that for any real vector bundle $\xi$ over $X$ we can find a real 
vector bundle $\xi^{\prime}$ such that $\xi \oplus \xi^{\prime}$ is a trivial bundle. This implies ( $X$ being connected) that any $x \in \widetilde{K O}(X)$ can be written in the form $\xi-n$ where $\xi$ is a real vector bundle with fibre $\mathbf{R}^{n}$ (or rather the corresponding element of $K O(X)$ ) and where $n$ is the trivial bundle with fibre $\mathbf{R}^{n}$ (i.e. $n$ times the unit of $K O(X)$ ). We write 'formally'

$$
w(x)=w(\xi)=\prod_{i=1}^{n}\left(1+x_{i}\right), \quad w(\rho)=\prod_{j=1}^{8}\left(1+y_{j}\right)=1+g .
$$

We calculate from now on simply in the cohomology ring of $S^{8} \times X$, i.e. we replace in the usual way certain tensor products of cohomology classes by cup-products. In $H^{*}\left(S^{8} \times X, Z_{2}\right)$ we have

$$
w\{\beta(x)\}=w\{(\rho-8) \otimes(\xi-n)\}=w(\rho \otimes \xi)\{w(\rho)\}^{-n}\{w(\xi)\}^{-8} .
$$

By (9) above and $\S 3$ we get, taking into account that the elementary symmetric functions of the $y_{j}$ vanish in positive degrees less than 8 ,

By (10) and (11) we get

$$
w(\rho \otimes \xi)=\Pi\left(1+x_{i}+y_{j}\right)=\prod_{i=1}^{n}\left\{\left(1+x_{i}\right)^{8}+g\right\} .
$$

$$
w\{\beta(x)\}=(1+g)^{-n} \prod_{i=1}^{n}\left(1+x_{i}\right)^{-8} \prod_{i=1}^{n}\left\{\left(1+x_{i}\right)^{8}+g\right\} .
$$

Remembering that we are calculating $\bmod 2$ and that $g^{2}=0$ we get

which proves the proposition because

$$
\begin{aligned}
w\{\beta(x)\} & =(1+n g) \prod_{i=1}^{n}\left(1+\frac{g}{1+x_{i}^{8}}\right), \\
& =1+n g+g \sum_{i=1}^{n}\left(1+x_{i}^{8}\right)^{-1},
\end{aligned}
$$

$$
\left(1+x_{i}^{8}\right)^{-1}=\sum_{k=0}^{\infty} x_{i}^{8 k}
$$

\section{REFERENCES}

(1) Aтtyan, M. F. and Hrrzerruch, F. Riemann-Roch theorems for differentiable manifolds. Bull. Amer. Math. Soc. 65 (1959), 267-81.

(2) Atryah, M. F. and Hirzebruch, F. Vector bundles and homogeneous spaces. Symposium on Differential Geometry, Tucson, Arizona, February 1960 (to appear).

(3) Borel, A. and Hrrzerruch, F. Characteristic classes and homogeneous spaces I, II. Amer. J. Math. 80 (1958), 458-538; 81 (1959), 315-82.

(4) Botr, R. The space of loops on a Lie group. Mich. Math. J. 5 (1958), 35-61.

(5) Botr, R. The stable homotopy of the classical groups. Ann. Math. 70 (1959), 313-37.

(6) Botr, R. Some remarks on the periodicity theorems. Colloque de Topologie, Lille, 1959 Bull. Soc. math. Fr. 87 (1959), 293-310.

(7) Botr, R. and Mrnor, J. On the parallelizability of the spheres. Bull. Amer. Math. Soc. 64 (1958), 87-9.

(8) Kervaire, M. A. Non-parallelizability of the $n$-sphere for $n>7$. Proc. Nat. Acad. Sci., Wash., 44 (1958), 280-3.

(9) Mrinor, J. Some consequences of a theorem of Bott. Ann. Math. 68 (1958), 444-9.

Pembroke College Cambridge
MATHEMatisches INSTITUT DER UNIVERSITÄT BONN 